This is the final peer-reviewed accepted manuscript of:

D. Cornigli et al.,

"Characterization of dielectric properties and conductivity in encapsulation materials with high insulating filler contents"

in IEEE Transactions on Dielectrics and Electrical Insulation, vol. 25, no. 6, pp. 24212428, Dec. 2018

The final published version is available online at:

https://doi-org.ezproxy.unibo.it/10.1109/TDEl.2018.007377

Rights / License:

The terms and conditions for the reuse of this version of the manuscript are specified in the publishing policy. For all terms of use and more information see the publisher's website.

This item was downloaded from IRIS Università di Bologna (https://cris.unibo.it/)

When citing, please refer to the published version. 


\title{
Characterization of dielectric properties and conductivity in encapsulation materials with high insulating filler contents
}

\author{
Davide Cornigli, Susanna Reggiani ${ }^{\star}$, Antonio Gnudi ${ }^{*}$ Elena Gnani ${ }^{\star}$, Giorgio Baccarani*, \\ Davide Fabiani \\ University of Bologna \\ Dept. of Electronics (DEI) and *Advanced Research Center on Electronic Systems "E. De Castro" \\ Bologna , Italy
}

\author{
Dhanoop Varghese, Enis Tuncer, Srikanth Krishnan and Luu Nguyen§ \\ Texas Instruments Inc. \\ Dallas, TX 75243, USA \\ ${ }^{\S}$ Santa Clara, CA 95051, USA
}

\begin{abstract}
The properties of different molding-compound materials with high filler contents have been investigated in order to assess their electrical properties. The experimental part of the present work has been focused on dielectric spectroscopy and steady-state conduction measurements. The results have been used to investigate the electrical properties of the materials at different frequencies, temperatures and electric fields. Differences in the relaxation kinetics with increasing filler content have been found, which can be ascribed to the larger interface regions between the filler particles. In addition, the extracted conductivities show a hopping transport and different activation energies on the temperature range from $20^{\circ} \mathrm{C}$ to $190^{\circ} \mathrm{C}$.
\end{abstract}

Index Terms - Spectroscopy, Epoxy resins, Dielectric polarization.

\section{INTRODUCTION}

EPOXY molding compounds (EMCs) are widely used in microelectronics for the encapsulation of integrated circuits, providing mechanical stability, protection from external environment and electrical insulation. With increasing device operating voltages and temperatures, the package dielectric materials are subjected to electrical and thermal stresses which could be detrimental for their reliability, and the study of their electrical properties becomes significantly important [1]. In particular, the characterization of EMCs in high stress environments gives useful information concerning failure mechanisms and reliability expectations [2]. In order to reduce the level of thermal expansion and to improve their mechanical stability, epoxy resins are usually filled with micro- and nano-sized particles: their content in commercial EMCs amount to about 65$90 \%$ by weight [3-4]. Micro and nanoparticles interact with the polymer matrix, influencing significantly the mechanical and electrical characteristics of the material. In this work, the electrical properties of two molding compounds filled with different contents of micro-sized silica particles have been investigated by means of dielectric spectroscopy and steady-state current measurements. The dielectric spectroscopy analysis (DEA) is usually employed to assess the properties of dielectrics [5], while steady-state current measurements could be used to identify the relation between the current and the electric field, giving information about the transport mechanisms. This work is organized as follows. The experimental setup and the measurement techniques are introduced in Section 2 while the experimental results are presented and discussed in Section 3 .

\section{EXPERIMENTS}

\subsection{MATERIALS}

In this study two different molding compounds based on the same epoxy matrix have been investigated. These encapsulation materials are thermoset-based polymeric composites, which have been obtained from Texas Instruments Incorporation. No specific interpretation of the type of resin has been carried out in this work as the detailed chemical formulation was not shared. The EMCs are labeled MC0 and $\mathrm{MC} 1$ and contain different fractions of silica fillers. The filler content was measured by thermogravimetric analysis (TGA) to determine the weight percentage, while the filler volume fraction has been 
Table 1. Properties of the mold compounds studied in this work

\begin{tabular}{c|c|c}
\hline Material label & Filler content & Filler volume \\
\hline MC0 & $91 \mathrm{wt} \%$ & $85 \%$ \\
\hline MC1 & $73 \mathrm{wt} \%$ & $59 \%$ \\
\hline
\end{tabular}

calculated from the densities of the epoxy matrix and fillers by using the mean size of filler particles. Measurements have been carried out on $50 \mathrm{~mm} \times 50 \mathrm{~mm}$ square samples with a thickness of $0.75 \mathrm{~mm}$. Dielectric strength, spectroscopy and DC conductivity experiments have been carried out on different sets of samples and different electrode configurations as explained in the next Sections. The properties of the molding compounds are summarized in Table 1.

\subsection{DIELECTRIC STRENGTH}

The dielectric strength measurements have been carried out by placing specimens in a mineral oil vessel, using spherical electrodes with $13 \mathrm{~mm}$ diameter as specified in IEC 60243-1 standards. An increasing AC voltage $(50 \mathrm{~Hz})$ has been applied, with a ramp rate of $1000 \mathrm{~V} / \mathrm{s}$ at $20^{\circ} \mathrm{C}$.

\subsection{DIELECTRIC ANALYSIS}

In order to determine the dielectric response of the materials, circular gold electrodes with $40 \mathrm{~mm}$ diameter were sputtered on both sides of the samples. After a preliminary bake in a thermostatic oven at $125^{\circ} \mathrm{C}$ for 24 hours to remove any residual moisture and polarization, dielectric spectroscopy analysis was carried out by using the Novocontrol Concept 80 alpha analyzer interfaced with the Novocontrol temperature control module in Nitrogen atmosphere. By applying a small signal with $\mathrm{V}_{\mathrm{RMS}}=1$ $\mathrm{V}$, the complex dielectric function $\varepsilon^{*}$ has been measured in the frequency range from $10^{2} \mathrm{~Hz}$ to $10^{6} \mathrm{~Hz}$, at different ambient temperatures. Two distinct measurement runs have been carried out on two different samples of each material.

\subsection{CURRENT ANALYSIS}

In order to study the relationship between the conductivity and the electric field, the steady-state current densities have been analyzed at temperatures ranging from $25{ }^{\circ} \mathrm{C}$ to $120^{\circ} \mathrm{C}$, corresponding to the maximum operating temperature of the experimental setup. The currents have been monitored in time with a Keithley-6514 electrometer for $7000 \mathrm{~s}$ during different DC step voltages into a thermostatic oven. The high-voltage electrode used in the measurements is circular with a diameter of $30 \mathrm{~mm}$, while the lower electrode has a diameter of $20 \mathrm{~mm}$ surrounded by a guard ring. Samples have been gold coated replicating the shapes of electrodes and guard ring.

Two distinct measurements have been carried out on a single sample of each material. Before each conductivity measurement, the samples have been short-circuited at $100{ }^{\circ} \mathrm{C}$ for 2 hours to remove any residual polarization.
Table 2. Weibull parameters ( $\alpha$ and $\beta)$, and correlation coefficient $\left(r^{2}\right)$ for the various materials tested.

\begin{tabular}{r|c|c|c}
\hline Material & $\boldsymbol{\alpha}[\mathbf{k V} / \mathbf{m m}]$ & $\boldsymbol{\beta}$ & $\mathbf{r}^{\mathbf{2}}$ \\
\hline MC0 & 36.7 & 14.3 & 0.98 \\
\hline MC1 & 38.4 & 18.0 & 0.94 \\
\hline
\end{tabular}

\section{RESULTS AND DISCUSSION}

In this section the experimental results are presented and discussed. The analysis starts with the characterization of the materials under breakdown $\mathrm{AC}$ tests, used to obtain the maximum electric field for each EMC. The investigation of the electrical properties is carried out by means of dielectric spectroscopy and DC current measurements at high electric fields. The conductivity is finally extracted and discussed for both materials.

\subsection{DIELECTRIC STRENGTH}

Four different breakdown measurements have been carried out on three different samples of each material. The results have been analyzed using a two-parameters Weibull cumulative probability function:

$$
P(E)=1-\exp \left[-(E / \alpha)^{\beta}\right]
$$

where $E$ is the experimentally recorded breakdown filed, $\alpha$ and $\beta$ are fitting parameters representing the characteristic breakdown strength and the shape parameter associated with the least-square linear fit of the distribution, respectively. The results are reported in Table 2, showing that the two materials have similar characteristic breakdown fields $\alpha$, indicating that the breakdown strength is unaffected by the filler content. This result is in agreement with the analysis reported in [6], where the influence of filler content on breakdown of epoxy resins has been investigated.

\subsection{DIELECTRIC ANALYSIS}

The dielectric spectroscopy is a powerful tool for the investigation of different physical processes such as the fluctuation of molecular dipoles, the charge transport and the separation of charges at interfaces. In general, in order to identify and quantify their contributions, the complex relative permittivity $\varepsilon^{*}$ as a function of the angular frequency $\omega$, is analyzed. More specifically, $\varepsilon^{*}(\omega)=\varepsilon^{\prime}(\omega)+\mathrm{i} \varepsilon^{\prime \prime}(\omega)$ is measured where $\varepsilon^{\prime}$ and $\varepsilon$ ' are the real permittivity and the dielectric loss factor, respectively. The analysis of these functions generally allows to extract the relaxation times of different dielectric processes and the conductivity contribution. In Figure 1, the real permittivity measured at temperatures between $20^{\circ} \mathrm{C}$ and $190{ }^{\circ} \mathrm{C}$ is reported for the two molding compounds. It is expected that the permittivity of the composites depends on the permittivity of each component, epoxy matrix and filler, with a linear trend with the filler volume fraction. A very limited variation of permittivity is 

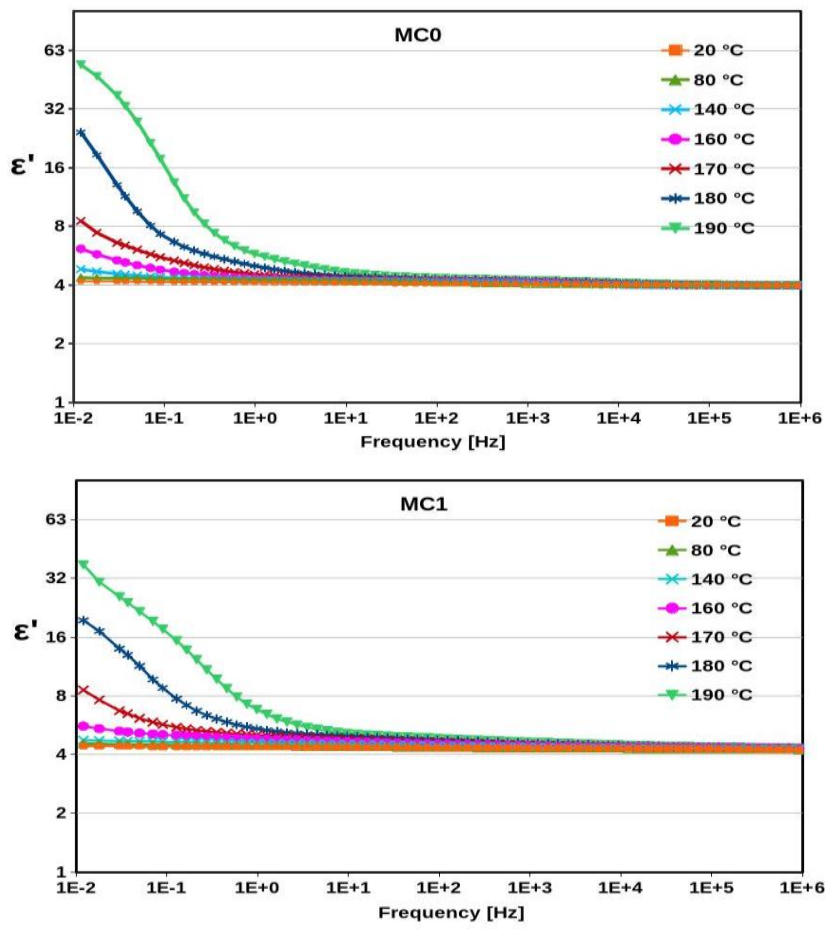

Figure 1. (Top) Real part of the dielectric permittivity of MC0 measured between $20^{\circ} \mathrm{C}$ and $190^{\circ} \mathrm{C}$. The strong increase of $\varepsilon$ ' at low frequencies and high temperatures could be ascribed to the presence of polarization processes such as Maxwell-Wagner-Sillars polarization [7]. (Bottom) The dielectric permittivity of $\mathrm{MC} 1$ shows a similar trend.

found for the two materials as the permittivity of the silica fillers is similar to that of the epoxy resin.. It can be noted that, at temperatures higher than $160{ }^{\circ} \mathrm{C}$, there is a strong increase of the permittivity at frequencies ranging from $10^{-2} \mathrm{~Hz}$ to $1 \mathrm{~Hz}$, which could indicate the onset of a relaxation process such as Maxwell-Wagner-Sillars (MWS) polarization [7-10]. This kind of polarization process is caused by the space-charge build-up at the interfaces between components with different properties. In molding compounds, the MWS relaxation is always present because of the fillers, and polarization due to space charge takes place at the mesoscopic epoxy/filler interfaces [11].A slightly larger increase of the MWS polarization effects is found for the EMC with larger filler content. In Figure 2, the dielectric loss of $\mathrm{MC} 0$ and $\mathrm{MC} 1$ is shown in the same range of temperatures. As expected, the imaginary part of the permittivity allows for the extraction of the conductivity contribution at low frequencies and shows the additional loss peaks related to the different relaxation processes at higher frequencies. The conductivity term at very low frequencies is characterized by the slope equal to -1 indicating an ohmic electrode behavior. In this regime, the conductivity $\sigma$ is proportional to the dielectric losses $\varepsilon " '$ :

$$
\sigma=\omega \varepsilon_{0} \varepsilon^{\prime \prime}(\omega)
$$

where $\varepsilon_{0}$ is the vacuum permittivity. The conductivity of $\mathrm{MC} 0$ and $\mathrm{MC} 1$ has been extracted at $10^{-2} \mathrm{~Hz}$ at temperatures greater than $160{ }^{\circ} \mathrm{C}$ by using Equation 2. The results are reported in Figure 3 as a function of the reciprocal temperature,
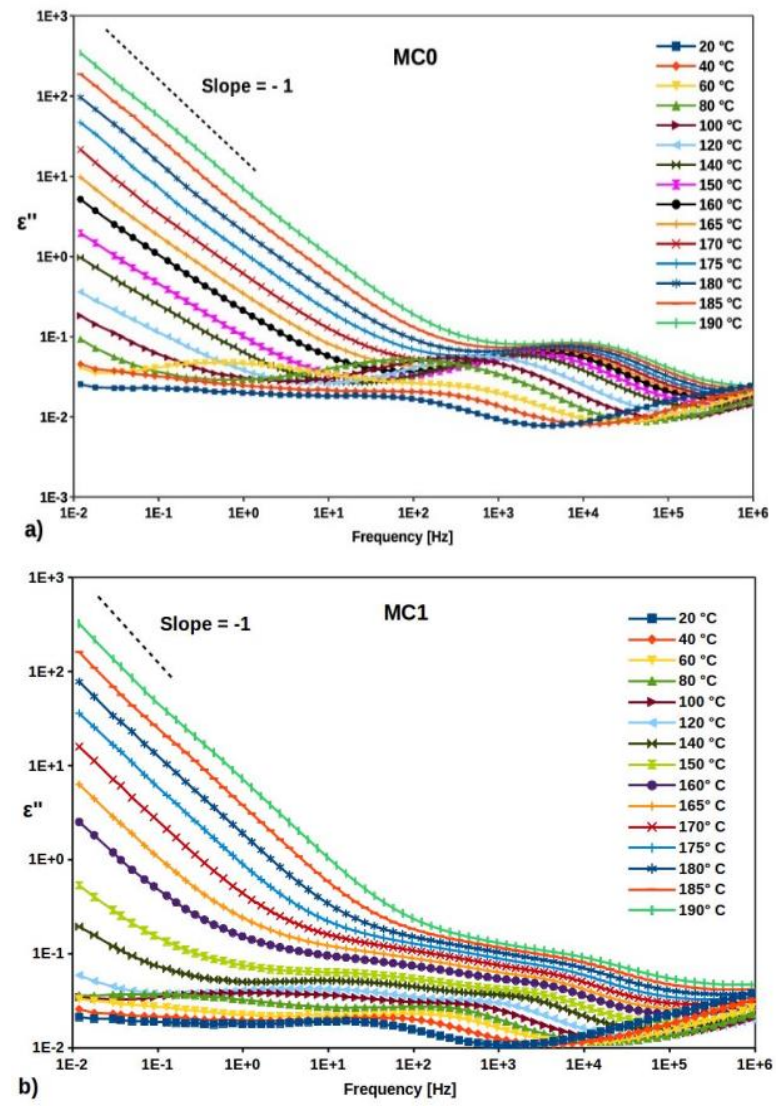

Figure 2. Imaginary part of the dielectric permittivity of $\mathrm{MC} 0$ (top) and $\mathrm{MC} 1$ (bottom) for temperatures from $20{ }^{\circ} \mathrm{C}$ to $190{ }^{\circ} \mathrm{C}$. The black dashed lines represent the slope of a purely ohmic conductivity term: deviations of the experimental data from such an ideal case indicate the presence of relaxation processes such as electrode polarization.

showing that $\sigma$ follows an Arrhenius-like trend:

$$
\sigma(T)=\sigma_{0} \exp \left(\frac{E_{a}}{k T}\right)
$$

where $\sigma_{0}$ is the pre-exponential term, $\mathrm{E}_{\mathrm{a}}$ is the activation energy in $\mathrm{eV}, \mathrm{k}$ is the Boltzmann constant and $\mathrm{T}$ is the temperature in Kelvin. Activation energies of $2.47 \mathrm{eV}$ and $2.84 \mathrm{eV}$ have been

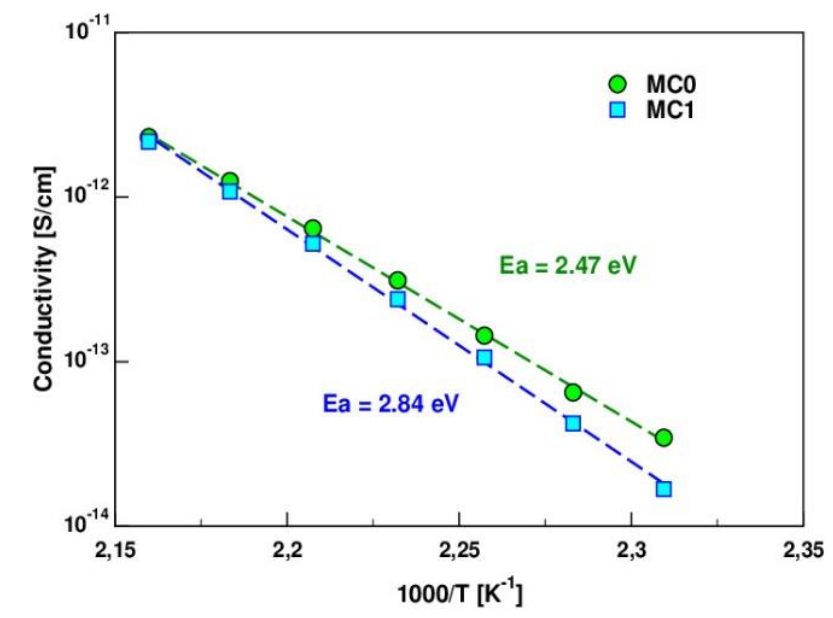

Figure 3. Conductivity of $\mathrm{MC} 0$ and $\mathrm{MC} 1$ extracted from dielectric losses at $10^{-2} \mathrm{~Hz}$ for temperatures $\mathrm{T}>160^{\circ} \mathrm{C}$ 

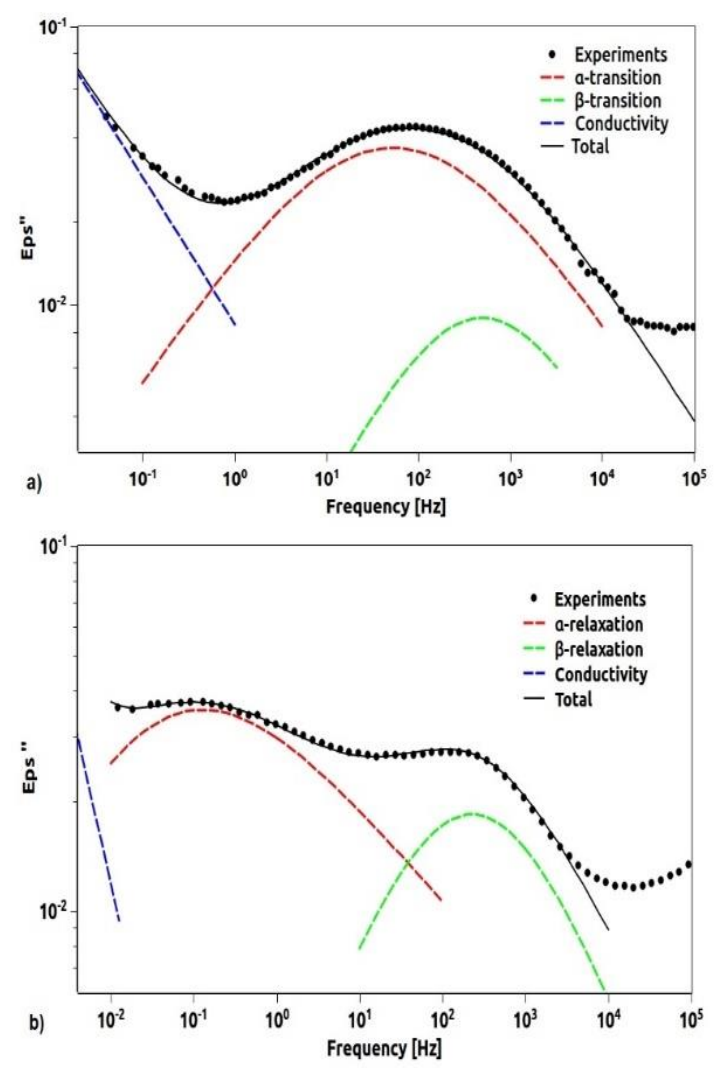

Figure 4. Deconvolution of the dielectric loss spectrum of MC0 (top) and $\mathrm{MC} 1$ (bottom) at $80{ }^{\circ} \mathrm{C}$.

obtained for the MC0 and MC1 samples, respectively.

The conductivity of $\mathrm{MC} 0$ is larger than the $\mathrm{MC} 1$ one on the whole temperature range, indicating an increase of conductivity with filler content, which is consistent with the analysis reported in [12]. The increase of conductivity with filler content was explained by the fact that a finite interfacial region around the fillers may be characterized by a different mobility.

At large frequencies, different relaxation processes can be identified in $\varepsilon "$, showing one or two relaxation peaks depending on the analyzed temperature regime. At relatively high temperatures, losses are due to the dipole relaxation, usually called $\alpha$ relaxation, which is related to the material viscosity [13]. The second one is visible at lower temperatures and is more evident for the material with the lower filler content, as shown in Figure 2b. This process could be related to the presence of fillers which make the system heterogeneous.

In order to investigate the properties of these relaxations, dielectric spectroscopy data $\varepsilon^{\prime}(\omega)$ and $\varepsilon^{\prime \prime}(\omega)$ have been simultaneously fitted by expressing $\varepsilon^{*}(\omega)$ as a sum of two different Havriliak-Negami (HN) functions and a conductivity term [14]:

$$
\varepsilon^{*}(\omega)=\varepsilon_{\infty}+\sum_{n=1}^{2} \frac{\Delta \varepsilon_{n}}{\left(1+\left(l \omega \tau_{n}\right)^{\alpha_{n}}\right)^{\beta_{n}}}-l \frac{\sigma}{\varepsilon_{0} \omega^{s}}
$$

with $\omega$ the angular frequency, $\varepsilon_{\infty}$ the unrelaxed dielectric permittivity, $\Delta \varepsilon_{\mathrm{n}}$ the dielectric relaxation strength, $\tau_{\mathrm{n}}$ the
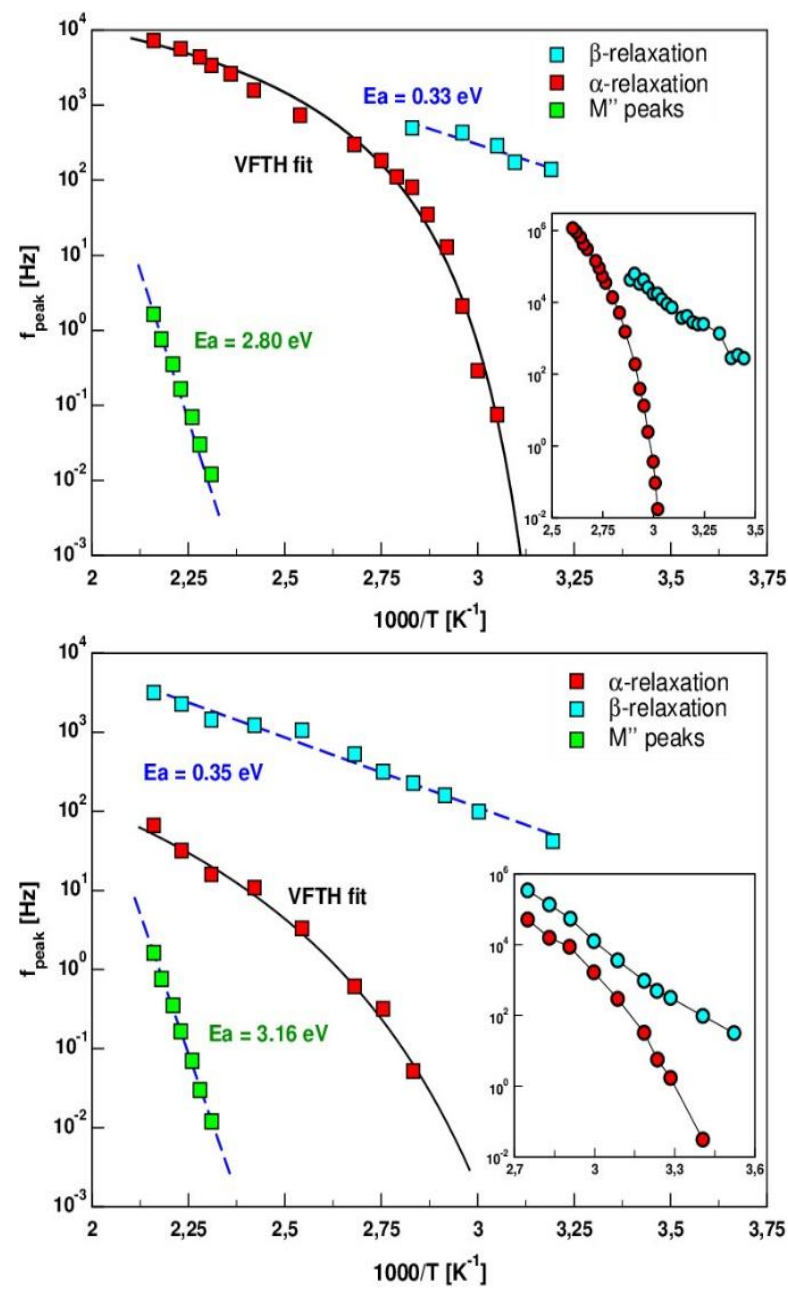

Figure 5. Reciprocal temperature dependence of the dielectric loss and modulus peak frequencies for MC0 (top) and MC1 (bottom). The inset in the top figure shows the data for PMMA reported in [15], while the inset in the bottom figure shows the data for PnBMA reported [16].

relaxation time, $\sigma$ the $\mathrm{DC}$ conductivity, s a fitting parameter, $\varepsilon_{0}$ the vacuum permittivity, $\alpha_{\mathrm{n}}$ and $\beta_{\mathrm{n}}$ the HN parameters. As an example, the deconvolution of the dielectric loss spectrum of $\mathrm{MC} 0$ and $\mathrm{MC} 1$ at $80^{\circ} \mathrm{C}$ is reported in Figure 4, highlighting the contributions given by $\alpha$ and $\beta$ relaxations to $\varepsilon$ ". By applying this fitting procedure, the characteristic frequencies $f_{\text {peak }}=\left(\begin{array}{lll}2 & \pi & \tau_{n}\end{array}\right)^{-1}$ associated to the HN functions have been determined from the dielectric losses at each temperature and their reciprocal temperature dependence has been reported in Figure 5. For MC0, one peak is present at temperatures up to 60 ${ }^{\circ} \mathrm{C}$, while a second one appears at $\mathrm{T}>60{ }^{\circ} \mathrm{C}$ and merges with the first, thus a single peak can be identified at temperatures higher than $80^{\circ} \mathrm{C}$. A similar trend has been observed in large number of polymers as, e.g., the PMMA reported in the inset of Figure 5a, showing a merging of the $\alpha$ and $\beta$ relaxations at higher temperatures [15]. The situation is quite different for $\mathrm{MC1}$, where two peaks can be clearly distinguished up to $180{ }^{\circ} \mathrm{C}$, showing a separate onset of the alpha regime, i.e., the $\alpha$ and $\beta$ transitions coexist up to temperatures greater than $T_{g}$. This behavior is similar to the one shown by the PnBMA material analyzed in [16] as shown in the inset of Figure 5b.

Differently, the low temperature process shows similar peak 

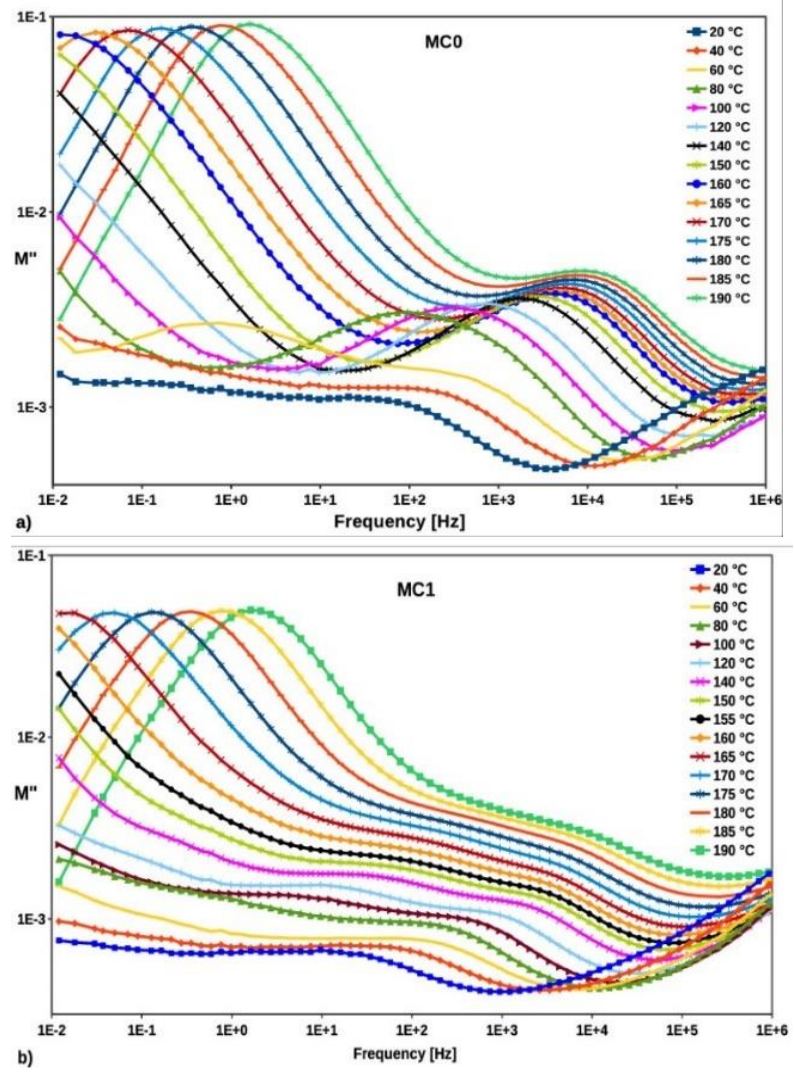

b)

Figure 6. Imaginary part of the electrical modulus of MC0 (top) and MC1 (bottom) measured between $20^{\circ} \mathrm{C}$ and $190{ }^{\circ} \mathrm{C}$.

frequencies for both molding compounds and can be described by an Arrhenius law leading to $\beta$-relaxation processes with activation energies of $0.33 \mathrm{eV}$ and $0.35 \mathrm{eV}$ for $\mathrm{MC} 0$ and $\mathrm{MC} 1$, respectively. The latter behavior is expected to be correlated mostly with the epoxy matrix as it is independent of the filler content. A similar behavior was reported in [17], showing very similar $\beta$-relaxation processes for pure epoxy and epoxy microcomposites. As explained in [17], since the molecular motion which originate the beta-relaxation lie along the polymeric chains of the epoxy, they are far away from the particles' interfaces and are not significantly affected by their presence. The $\alpha$-relaxation process at high temperatures is nicely described by the Vogel-Fulcher-Tammann-Hesse (VFTH) equation [18-20]:

$$
f_{\text {peak }}(T)=f_{\infty} \exp \left(-\frac{B}{T-T_{0}}\right)
$$

where $\mathrm{f}_{\infty}$ and $\mathrm{B}$ are fitting constants, while $\mathrm{T}_{0}$ denotes the Vogel temperature. The parameters obtained by fitting the data with Equation 5 are shown in Table 3. The glass transition temperatures $T_{g}$ have been calculated from the interpolation value $\log _{10}\left(2 \pi f_{\text {peak }}\right)=-2$. It can be noted that the $\mathrm{MC} 1$ shows a $\mathrm{T}_{\mathrm{g}}$ which is about $11^{\circ} \mathrm{C}$ greater than $\mathrm{MC} 0$, suggesting that an higher the filler content corresponds to a lower glass transition temperature. The $\alpha$-relaxation peak frequencies are significantly different in the two materials, as can be noted from the parameter $f_{\infty}$ which varies about one order of magnitude passing from $\mathrm{MC} 0$ to $\mathrm{MC}$. The latter process is thus expected to be correlated to the filler content and/or the filler/epoxy interfaces. A similar modification in the $\alpha$-relaxation between samples of pure epoxy and micro-composites was observed also in [17] even if the characteristic values are not comparable to those reported in this work: while $T_{0}$ showed a decrease with fillers, the B parameter is significantly increased. The latter features were explained by the fact that the mobility of the macromolecules is enhanced (as the macromolecules are shorter) even if the movements are hindered by a larger potential barrier.

A third relaxation contribution is expected in the dielectric loss at high temperature and low frequency due to the MWS polarization clearly observed from the real permittivity curves. Both materials show a strong conductivity contribution which adds to the MWS polarization making it difficult to identify the corresponding relaxation peaks. In order to investigate this feature, the complex electric modulus $M^{*}(\omega)=M^{\prime}(\omega)+i M^{\prime \prime}(\omega)$, which is defined as the inverse of the complex relative permittivity, has been analyzed. This quantity is simply an alternative representation of the dielectric properties of a material, showing the tendency to strongly limit or eliminate the role of large loss values due to conductivity overwhelming relaxation peaks in the dielectric spectrum [21]. As shown in Figure 6, the imaginary part of the modulus exhibits welldefined peaks at temperatures higher than $150{ }^{\circ} \mathrm{C}$, which shift towards higher frequencies as temperature increases. A similar trend has been observed in various insulating polymers and seems to be ascribed to the ionic hopping: at frequencies below the relaxation peak of M" charge carriers are mobile over long distances (quasi-ohmic regime), while at higher frequencies they are confined by higher potential barriers and their motion is inhibited [21-23]. This ionic long-range hopping is induced by long distance molecular motion, the relative relaxation process is thermally activated and closely related to the glass transition [22] and it is usually visible for $\mathrm{T}>\mathrm{T}_{\mathrm{g}}$. As reported in Figure 5, the relaxation peaks of $\mathrm{MC} 0$ and $\mathrm{MC} 1$ show similar values, indicating that this kind of processes are related mostly to the epoxy matrix. Furthermore, the modulus peaks show an Arrhenius reciprocal temperature dependence, corresponding to an activation energy of $2.80 \mathrm{eV}$ and $3.16 \mathrm{eV}$ for $\mathrm{MC} 0$ and $\mathrm{MC} 1$, respectively. These activation energies should be similar to the ones of the DC conductivity, being both mechanisms associated to ionic hopping transport, but a discrepancy between the two

Table 3. $\alpha$-relaxation parameters

\begin{tabular}{r|c|c|c|c}
\hline Material & $\mathbf{f}_{\infty}[\mathbf{H z}]$ & $\mathbf{B}[\mathbf{K}]$ & $\mathbf{T}_{\mathbf{0}}[\mathbf{K}]$ & $\mathbf{T}_{\mathrm{g}}\left[{ }^{\circ} \mathbf{C}\right]$ \\
\hline MC0 & $8.1 \times 10^{4}$ & 440 & 298 & 48 \\
\hline MC1 & $8.5 \times 10^{3}$ & 992 & 296 & 59 \\
\hline
\end{tabular}


Table 4. Shape parameters obtained by using Equation 5.

\begin{tabular}{r|c|c}
\multicolumn{3}{|c}{$\boldsymbol{\beta}$-parameter } \\
\hline T $\left[{ }^{\circ} \mathbf{C}\right]$ & MC0 & MC1 \\
\hline 170 & 0.83 & 0.83 \\
\hline 180 & 0.83 & 0.86 \\
\hline 190 & 0.84 & 0.85 \\
\hline
\end{tabular}

mechanisms of about $0.3 \mathrm{eV}$ has been found for both materials. This difference can be explained by considering that an accurate extraction of the ohmic conductivity from the dielectric losses would require measurements at frequencies lower than $10^{-2} \mathrm{~Hz}$. In order to further investigate the dielectric modulus peaks, the Cole-Cole relaxation model has been used:

$$
M^{\prime \prime}(\omega)=\frac{\Delta M}{1+\left(l \omega \tau_{M}\right)^{\beta}}
$$

where the parameters $\Delta \mathrm{M}$ and $\tau_{\mathrm{M}}$ depend on the static and unrelaxed dielectric permittivity $\varepsilon_{\mathrm{s}}$ and $\varepsilon_{\infty}$, respectively as shown in [21]. The shape parameter $\beta$ in the modulus format remains unchanged with respect to the permittivity one and is close to the unity when the process can be described by a single Debye relaxation. The experimental data have been fitted at temperatures higher than $165^{\circ} \mathrm{C}$ by using Equation 6 . In Table 4 , the extracted shape parameters are reported for $\mathrm{MCO}$ and $\mathrm{MC} 1$, showing a deviation from $\beta=1$ which confirms the presence of distributed relaxation processes in both materials.

\subsection{HIGH-FIELD CONDUCTION}

The DC current measurements have been carried out at electric fields ranging from $4 \mathrm{kV} / \mathrm{mm}$ to $20 \mathrm{kV} / \mathrm{mm}$. The DC conductivity can be extracted from the high-field current density if the steady-state regime is reached. To this purpose, the currents have been measured for $7000 \mathrm{~s}$ in order to distinguish between polarization and carrier conduction. As an example, the current curves as functions of the time evolution are reported in Fig. 7 for MC0 at three different temperatures, showing the current stability at $7000 \mathrm{~s}$ even at $20^{\circ} \mathrm{C}$.

The current density at $7000 \mathrm{~s}$ as a function of the electric field is reported in Fig. 8 for both molding compounds at $80^{\circ} \mathrm{C}, 100$ ${ }^{\circ} \mathrm{C}$ and $120^{\circ} \mathrm{C}$. At temperatures lower than $80^{\circ} \mathrm{C}$ measurements are very noisy and the increase of the currents due to the electric field is of the same order of magnitude of the experimental uncertainty, making it difficult to find meaningful results. Various high-field conduction theories have been proposed to describe the relationship between the current and the electric field in insulating polymers, such as Schottky emission (SE), Poole-Frenkel effect (PF) and ionic hopping conduction (IH) [24]. In the case of Schottky emission, a field-assisted thermionic injection from the electrode occurs and the current

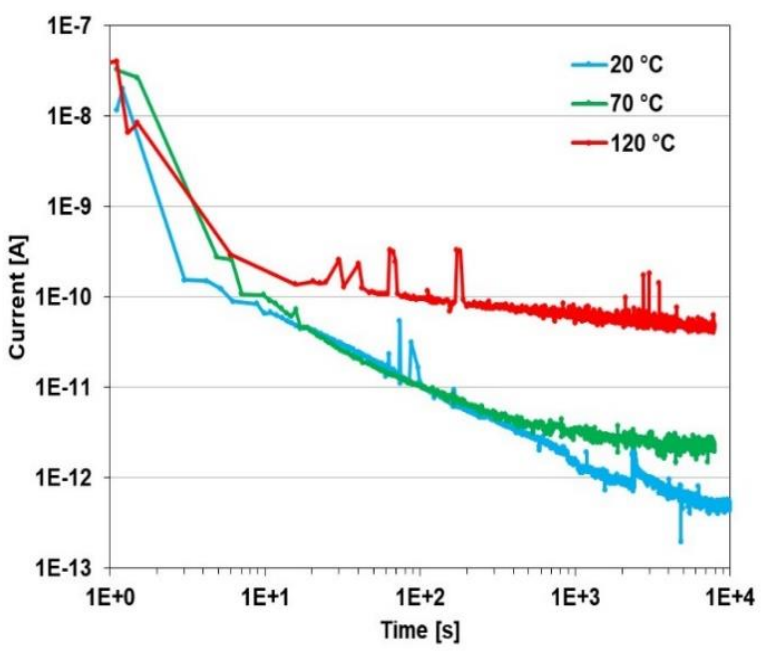

Figure 7. Time evolution of the currents of $\mathrm{MCO}$ after the application of a step voltage of $4 \mathrm{kV}$, measured at $20{ }^{\circ} \mathrm{C}, 70{ }^{\circ} \mathrm{C}$ and $120^{\circ} \mathrm{C}$. It can be noted that the curves are nearly flat after $7000 \mathrm{~s}$, even at $20^{\circ} \mathrm{C}$. $\mathrm{MC} 1$ shows similar results.

density $\mathbf{J}_{\mathrm{SE}}$ can be expressed as:

$$
\begin{aligned}
& J_{S E}=A T^{2} \exp \left(-\frac{\phi_{B}-\beta_{S} \sqrt{E}}{k T}\right) \\
& \beta_{S}=\left(\frac{q^{3}}{4 \pi \varepsilon_{r} \varepsilon_{0}}\right)^{1 / 2}
\end{aligned}
$$

where A is the effective Richardson constant, $\phi_{\mathrm{B}}$ the Schottky barrier height, $\mathrm{q}$ is the electron charge, $\varepsilon_{\mathrm{r}}$ is the relative permittivity and $\mathrm{k}$ is the Boltzmann constant.

The PF effect involves a mechanism which is very similar to the Schottky emission and the current density has again an exponential dependence from the square root of the electric field:

$$
\begin{aligned}
& J_{P F}=B E \exp \left(-\frac{\phi_{P F}-\beta_{P F} \sqrt{E}}{k T}\right) \\
& \beta_{P F}=\left(\frac{q^{3}}{\pi \varepsilon_{r} \varepsilon_{0}}\right)^{1 / 2}
\end{aligned}
$$

where $\mathrm{B}$ is a constant and $\phi_{\mathrm{PF}}$ is the trap energy level. In the case of ionic conduction, the relation between current density and electric field assumes the form [25]:

$$
J_{I H}=J_{0} \sinh \left(\frac{q \lambda E}{2 k T}\right)
$$

where $\mathrm{J}_{0}$ is the zero field current density and $\lambda$ is the hopping distance between adjacent sites for the ions. The ionic conduction is enhanced by the electric field as it lowers the barrier height between adjacent sites in one direction and increases it in the opposite direction, giving rise to a net flux 

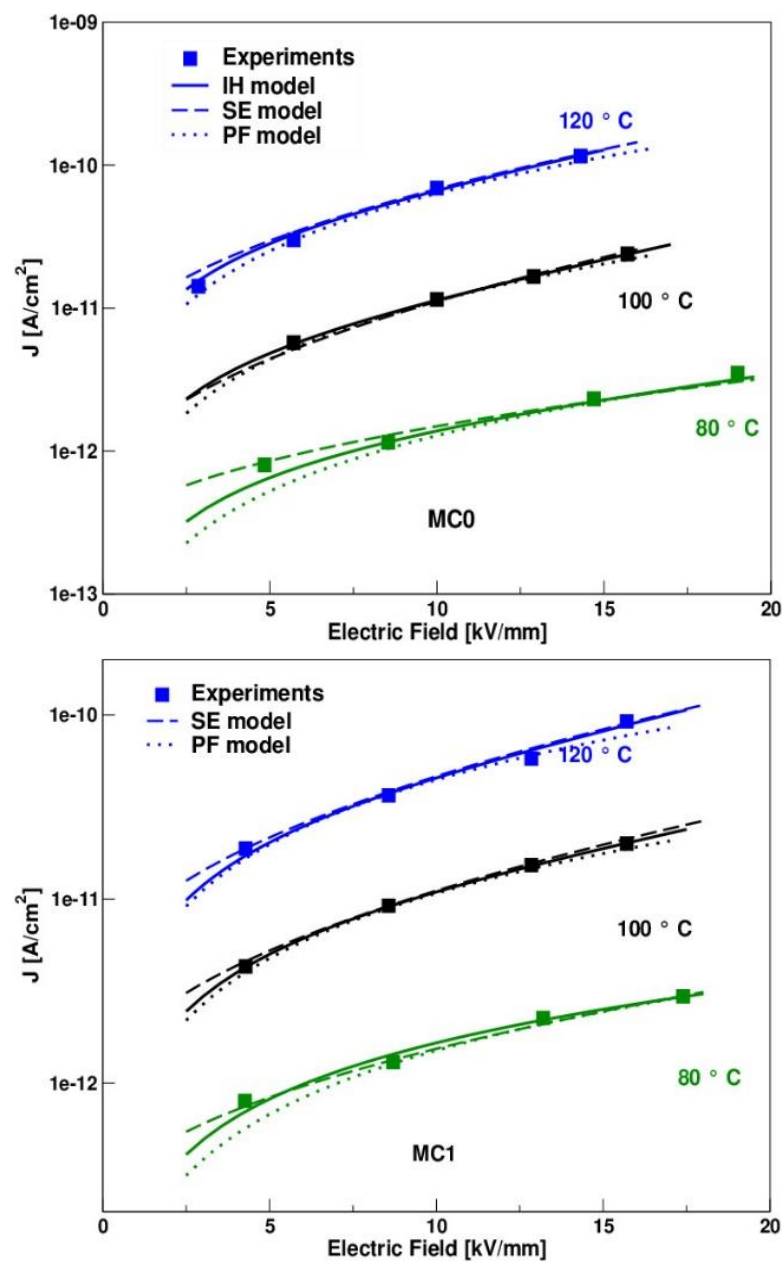

Figure 8. Current density as a function of electric field of the sample $\mathrm{MC} 0$ and $\mathrm{MC} 1$ at $80{ }^{\circ} \mathrm{C}, 100{ }^{\circ} \mathrm{C}$ and $120{ }^{\circ} \mathrm{C}$. Filled squares: experiments. Solid lines: ionic conduction model. Dotted lines: Poole-Frenkel model. Dashed lines: Schottky emission model.

following a sinh law, while an Arrhenius-like temperature dependence is expected in the coefficient $\mathbf{J}_{0}$.

From Equations 7 and 9 it follows that the relative permittivity $\varepsilon_{\mathrm{r}}$ of the material can be calculated from the slope of $\ln (J)-E^{1 / 2}$ and $\ln (J / E)-E^{1 / 2}$ curves in the case of Schottky emission and $\mathrm{PF}$ transport, respectively. The extracted $\varepsilon_{\mathrm{r}}$ for $\mathrm{MC} 0$ and $\mathrm{MC1}$ with the SE model are significantly different from the measured dielectric constant at high frequency. In fact, the permittivity measured with spectroscopy at $1 \mathrm{MHz}$ shows values between 4.0 and 4.2 for temperatures between $70{ }^{\circ} \mathrm{C}$ and $120{ }^{\circ} \mathrm{C}$ for both compounds, while the dielectric constant extracted with SE model strongly decreases with temperature, changing from $3.6(4.0)$ at $80^{\circ} \mathrm{C}$ to $1.2(1.8)$ at $120^{\circ} \mathrm{C}$ for $\mathrm{MC} 0$

Table 5. Hopping distance $\lambda$ extracted from IH model

\begin{tabular}{r|c|c}
\multicolumn{3}{c}{$\lambda$-parameter $[\mathrm{nm}]$} \\
\hline $\mathbf{T}\left[{ }^{\circ} \mathbf{C}\right]$ & MC0 & MC1 \\
\hline 80 & 4.4 & 1.6 \\
\hline 100 & 7.5 & 5.4 \\
\hline 120 & 7.9 & 6.6 \\
\hline
\end{tabular}

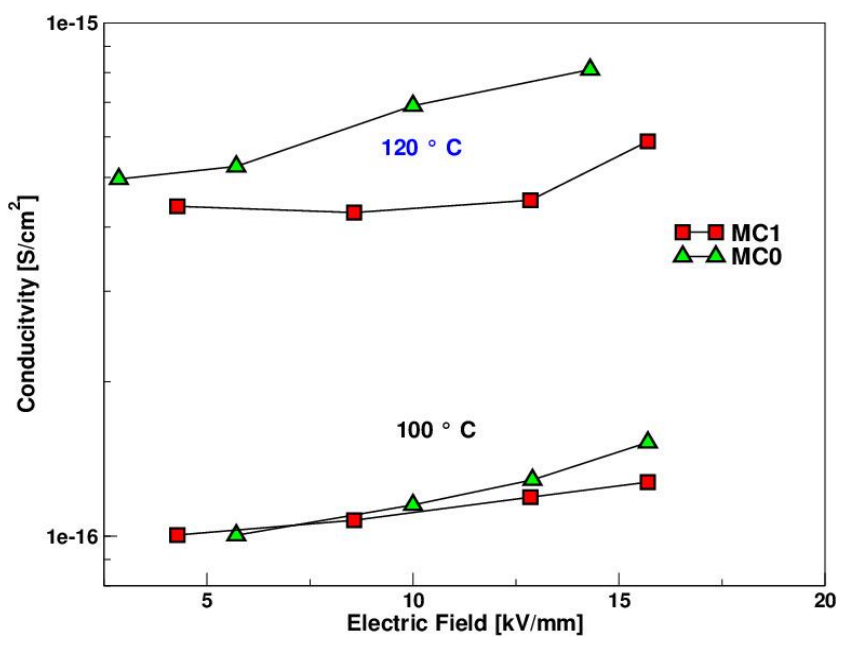

Figure 9. Electric field dependence of conductivity of $\mathrm{MC} 0$ and $\mathrm{MC} 1$ extracted from DC currents at $100{ }^{\circ} \mathrm{C}$ and $120^{\circ} \mathrm{C}$.

(MC1). Analogue considerations apply to the PF model, which leads to find extremely large values of $\varepsilon_{\mathrm{r}}$ and to fit the experiments with less precision, especially at $\mathrm{T}<100^{\circ} \mathrm{C}$. This leads to conclude that SE and PF models appear to describe the high-field current curves with a wrong $\varepsilon_{\mathrm{r}}$ especially at high temperatures. This could be ascribed to the accumulation of charge at the electrodes which alters the electric field inside the materials, and an accurate analysis of this behavior would require space charge measurements [26].

In order to further investigate the high-field conduction, a different transport process has been analyzed. From Equation (11), it is possible to obtain the value of the hopping parameter $\lambda$ by fitting $J$ as a function of $\sinh (E)$. The results are reported in Table 5, showing that $\lambda$ increases with temperature with values that are similar to those observed in various polymeric materials such as polyvinyl chloride, polyimide and parylene [25, 27-28]. In the previous works, this behavior has been qualitatively interpreted by assuming a temperature dependent height of the potential barriers which limits the ionic migration. The hopping distance exhibits a marked increase with temperature in the glass transition region and tends to saturate when the material is in the rubbery state [27].

The conductivity $\sigma$ of the EMCs can be extracted from steady state current measurements by using the relation:

$$
\sigma=\frac{I}{A E} \equiv \frac{I h}{A V}
$$

where $\mathrm{I}$ is the current, $\mathrm{E}$ is the electric field, $\mathrm{V}$ is the voltage between the electrodes, $\mathrm{A}$ is their area and $\mathrm{h}$ is the thickness of the sample. In Figure 9, the conductivities of MC0 and MC1 extracted at $100{ }^{\circ} \mathrm{C}$ and $120^{\circ} \mathrm{C}$ are reported as functions of the electric field. The larger amount of fillers in MC0 leads to a more effective field enhancement in the conductivity with respect to $\mathrm{MC1}$, which can be ascribed to a larger hopping distance as reported in Table 5. This feature could be due to 


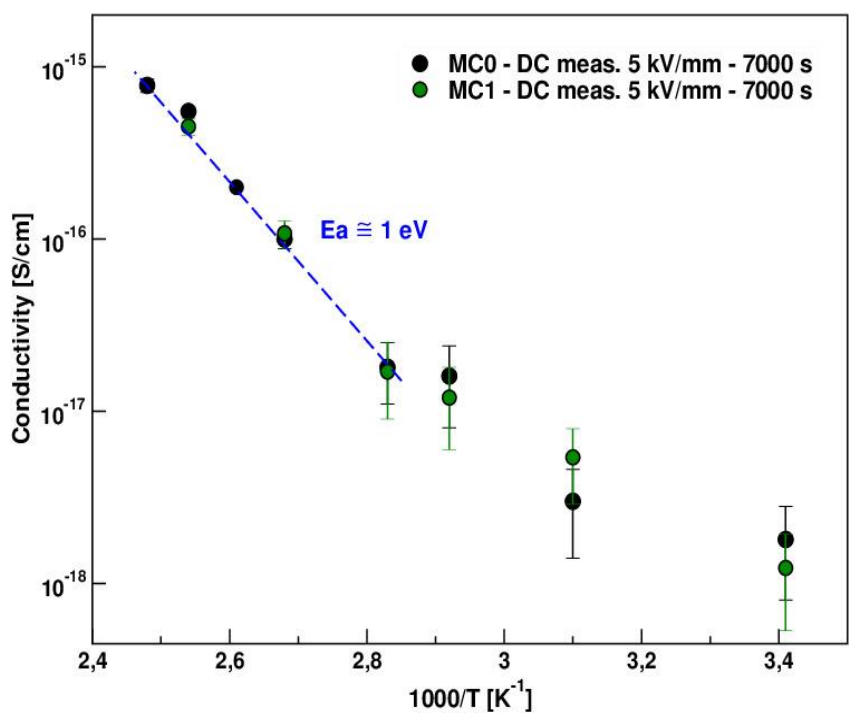

Figure 10. Reciprocal temperature dependence of DC conductivity of MC0 (black circles) and $\mathrm{MC1}$ (green circles) extracted from DC currents.

different properties of the filler/epoxy interfaces. respect to $\mathrm{MC} 1$, which can be ascribed to a larger hopping distance as reported in Table 5.

In order to investigate the temperature effects on charge transport at $\mathrm{T}<\mathrm{T}_{\mathrm{g}}$, the conductivities of $\mathrm{MC} 0$ and $\mathrm{MC} 1$ have been extracted at a field of about $5 \mathrm{kV} / \mathrm{mm}$ at temperatures ranging from $25{ }^{\circ} \mathrm{C}$ to $130{ }^{\circ} \mathrm{C}$. The reciprocal temperature dependence of $\sigma$ is shown in Figure 10. It should be noted that both materials show similar conductivities and temperature dependencies. At temperatures lower than $70{ }^{\circ} \mathrm{C}$ very small currents have been measured presenting a significant noise. The corresponding conductivity could be ascribed to the presence of a small amount of ionic impurities into the materials. The activation energy has been thus extracted for $\mathrm{T}>80^{\circ} \mathrm{C}$ and both materials show a similar activation energy of about $1 \mathrm{eV}$. By comparing Figure 9 and Figure 10, an increase of conductivity for $\mathrm{MC} 0$ is expected at larger temperatures due to the thermallydriven enhancement. By comparing Figures 3 and 10, a significant increase of the activation energy at higher temperatures has been found for both materials. Above the glass transition temperature, the occurrence of segmental motion results in an increase of the free volume which, in turn, facilitates the motion of ionic charge carriers [29].

\section{CONCLUSIONS}

The role of filler content in two different EMCs commercially used for the encapsulation of integrated circuits has been characterized by means of breakdown analysis, dielectric spectroscopy and steady-state current measurements in a wide range of temperatures. No significant effects on the breakdown have been observed even if dielectric properties and conductivities are affected by the filler volume fraction. In particular, at high temperatures an increase in the filler quantity gives a larger conductivity and an enhanced electric field dependence. This behavior could be due to the larger interface regions between the filler particles and the epoxy matrix. The analysis carried out in this work is an essential starting point, even though a complete reliability characterization would also require long-term electro-thermal ageing studies.

\section{ACKNOWLEDGMENT}

This work was supported by Texas Instruments Inc., Dallas, TX.

\section{REFERENCES}

[1] A. Garcia, N. Warner, N. A. D’Souza, E. Tuncer, L. Nguyen, M. Denison, and J. T. Fong, "Reliability of high-voltage molding compounds:Particle size, curing time, sample thickness, and voltage impact on polarization," IEEE Transactions on Industrial Electronics, vol. 63,no. 11, pp. 71047111, Nov 2016.

[2] L. Lanz, S. Hwang, and M. Pecht, "Characterization of plastic encapsulant materials as a baseline for quality assessment and reliability testing," Microelectron. Reliab., vol. 42, pp. 1163-1170, 2002.

[3] D. Fabiani, G. C. Montanari, A. Krivda, L. E. Schmidt, and R. Hollertz, "Epoxy based materials containing micro and nano sized fillers for improved electrical characteristics," in 2010 10th IEEE International Conference on Solid Dielectrics, July 2010, pp. 1-4.

[4] P. Jae-Jun, "Effect of Silica Content on the Dielectric Properties of Epoxy/Crystalline Silica Composites," Trans. Electr. Ectron Mater, vol. 13, pp. 322-325, 2012.

[5] R. McKenzie, W. Zurawsky, and J. Mijovic, "A molecular interpretation of Maxwell-Wagner-Sillars processes,” J. of Non-Cryst. Solids, vol. 406, pp. 11-21, 2014.

[6] G. Iyer, R. S. Gorur, R. Richert, A. Krivda, and L. E. Schmidt, "Dielectric properties of epoxy based nanocomposites for high voltage insulation," IEEE Transactions on Dielectrics and Electrical Insulation, vol. 18, no. 3, pp. 659-666, June 2011.

[7] R. W. Sillars, "The Properties of a Dielectric Containing SemiConducting Particles of Various Shapes,” J. Inst. Elect. Eng., vol. 80, pp. 378-394, 1937.

[8] J. C. Maxwell, A Treatise on Electricity and Magnetism. Clarendon Press, Oxford, third edition. reprint by Dover, 1891.

[9] K. W. Wagner, Ann. Phys., 40, 817 (1913).

[10] K. W. Wagner Archiv. Electrotech, II, 371 (1914).

[11] E. Tuncer and G. A. Niklasson, "Properties of Bruggeman Dielectric Mixture Expression," in IEEE Conference on Electrical Insulation and Dielectric Phenomena, 2014, pp. 1-4.

[12] I. Imperiale, S. Reggiani, G. Pavarese, E. Gnani, A. Gnudi, G. Baccarani, W. Ahn, M. A. Alam, D. Varghese, A. Hernandez-Luna, L. Nguyen, and S. Krishnan, "Role of the Insulating Fillers in the Encapsulation Material on the Lateral Charge Spreading in HV-ICs," IEEE Trans. Electron Devices, vol. 64, pp. 1209-1216, 2017.

[13] H. Couderc, A. Saiter, J. Grenet, J. M. Saiter, G. Boiteux, E. Nikaj, I. Stevenson, and N. DSouza, "Relaxation Map of PETg-Montmorillonite Composites: Nanofiller Concentration Influence on. $\alpha$ and $\beta$ Relaxation Processes,” Polym. Eng. Sci., vol. 49, pp. 836-843, 2009.

[14] S. Havriliak and S. Negami, "A complex plane representation of dielectric and mechanical relaxation processes in some polymers," Polymer, vol. 8, pp. 161-210, 1967.

[15] L. Hartmann, W. Gorbatschow, J. Hauwede, and F. Kremer, "Molecular dynamics in thin films of isotactic poly(methyl methacrylate)," The European Physical Journal E, vol. 8, no. 2, pp. 145-154, May 2002.

[16] F. Garwe, A. Schonhals, M. Beiner, K. Schroter, and E. Donth, "Molecular cooperativity against locality at glass transition onset in poly(n butyl methacrylate)," Journal of Physics: Condensed Matter, vol. 6, no. 35, p. 6941, 1994.

[17] H. Couderc, M. Frechette, E. David, and S. Savoie, "Study of dielectric relaxation of epoxy composites containing micro and nano particles," IEEE Transactions on Dielectrics and Electrical Insulation, vol. 20, no. 2, pp. 592-600, April 2013.

[18] H. Vogel, Phys. Z, vol. 22, p. 645, 1921. 
[19] G. S. Fulcher, "Analysis of recent measurements of the viscosity of glasses," Journal of the American Ceramic Society, vol. 8, no. 6, pp. 339$355,1925$.

[20] W. H. G. Tammann, Z Anorg. Allg. Chem., vol. 156, p. 245, 1926.

[21] F. Tian and Y. Ohki, "Electric modulus powerful tool for analyzing dielectric behavior," IEEE Transactions on Dielectrics and Electrical Insulation, vol. 21, no. 3, pp. 929-931, June 2014.

[22] Y. Ohki, "Dielectric relaxation phenomena of several insulating polymers analyzed by electric modulus spectra," in 2015 IEEE 11th International Conference on the Properties and Applications of Dielectric Materials (ICPADM), July 2015, pp. 192-195.

[23] K. Abdelkader, R. Pascal, L. Olivier, and R. Cline, "Dielectric relaxation and ionic conduction in 66\% silica/cw229-3/hw229-1 microcomposite polymer," Composites Part B: Engineering, vol. 78, pp. 488 - 496, 2015.

[24] T. Mizutani, "High field phenomena in insulating polymers," in Proceedings of the 2004 IEEE International Conference on Solid Dielectrics, 2004. ICSD 2004., vol. 1, July 2004, pp. 11-16 Vol.1.

[25] G. G. Raju, R. Shaikh, and S. U. Haq, "Electrical conduction processes in polyimide films - I," IEEE Transactions on Dielectrics and Electrical Insulation, vol. 15 , no. 3, pp. 663-670, June 2008 .
[26] J. Castellon, H. N. Nguyen, S. Agnel, A. Toureille M. Fréchette, S. Savoie, A. Krivda and L.E. Schmidt "Electrical properties analysis of micro and nano composite epoxy resin materials," in IEEE Transactions on Dielectrics and Electrical Insulation, vol. 18, no. 3, pp. 651-658, June 2011.

[27] M. Kosaki, K. Sugiyama, and M. Ieda, "Ionic jump distance and glass transition of polyvinyl chloride," Journal of Applied Physics, vol. 42, no. 9, pp. 3388-3392, 1971.

[28] A. Kahouli, F. Jomni, A. Sylvestre, B. Yangui, and J. Legrand, "I - T , J$1 / \mathrm{T}$ and $\mathrm{j}-\mathrm{E}$ characteristics for the understanding of the main mechanism of electric conduction and the determination of the glass transition temperature of parylene c thin films," Journal of Physics D: Applied Physics, vol. 44, no. 50, p. 505302, 2011.

[29] T. Miyamoto and K. Shibayama, "Ionic conduction mechanism of polymers," in Conference on Electrical Insulation Dielectric Phenomena - Annual Report 1973, Oct 1973, pp. 431-438. 